\title{
CONTRACEPTIVOS DE EMERGÊNCIA: UMA REVISÃO TEÓRICA DO TIPO NARRATIVA PARA IDENTIFICAR EVIDÊNCIAS DA FARMACODINÂMICA E DO USO DESSES MEDICAMENTOS
}

\author{
EMERGENCY CONTRACEPTIVES: A NARRATIVE-TYPE THEORETICAL REVIEW \\ TO IDENTIFY EVIDENCE OF THE PHARMACODYNAMICS AND USE OF THESE \\ MEDICINES
}

\author{
Gabrielle Adorno Silva ${ }^{1}$ \\ Gabrielle Racoski Custódio Pillati² \\ Rafaela dal Piva ${ }^{3}$
}

RESUMO: Contraceptivo de emergência (CE), conhecidos popularmente como "pílula do dia seguinte" comercializados no Brasil, é um método que se distingue dos demais anticoncepcionais uma vez que este é utilizado após o ato sexual sem proteção, enquanto, os demais previnem a concepção antes e durante as relações sexuais. Este estudo teve como objetivo possibilitar ao leitor de forma clara e análise sobre seu uso e efeitos, desejáveis e indesejáveis, no organismo da mulher, devido ao uso recorrente desses contraceptivos. Para tanto, realizou-se uma revisão teórica do tipo narrativa alicerçada em pesquisas e trabalhos publicados entre os anos de 20II a 202I. Conclui-se com esse estudo que há necessidade de que as mulheres recebam maiores informações sobre indicação, métodos disponíveis, eficácia e segurança fundamentais a fim de evitar uma gravidez indesejada e que o uso de CE regularmente pode sim causar problemas de saúde. Espera-se que os

\footnotetext{
I Acadêmica de Farmácia pelo CESUFOZ - Centro de Ensino Superior de Foz do Iguaçu. E-mail: gabrielle_adorno@outlook.com

${ }^{2}$ Farmacêutica generalista pela Universidade Estadual do Oeste do Paraná - UNIOESTE (2013), mestre em Ciências Farmacêuticas pela UNIOESTE (2018), membro do corpo docente dos cursos de Farmácia e Biomedicina do Centro de Ensino Superior de Foz do Iguaçu - CESUFOZ.

${ }^{3}$ Graduação em Farmácia - Habilitação Indústria pela Universidade Estadual do Oeste do Paraná (2004). Mestrado em Biotecnologia Aplicada a Agricultura pela UNIPAR (2011). Experiência em farmácia de dispensação com assistência farmacêutica, elaboração de procedimentos operacionais padrão, controle de psicotrópicos. Experiência em manipulação de medicamentos alopáticos nas formas sólidas, líquidas e semissólidas; controle de qualidade de matérias-primas utilizadas em manipulação, elaboração de procedimentos operacionais padrão. Experiência na indústria farmacêutica na área da garantia da qualidade com elaboração de ordens de fabricação, liberação de produtos, conhecimentos do sistema SAP, elaboração de relatórios de revisão periódica de produtos, análise estatística de dados, conhecimentos de controle de mudanças e validação de processos e atuação na área de assuntos regulatórios pertinentes à pós registro de medicamentos e histórico de mudança de produtos. Atuação na docência em cursos técnicos nas disciplinas de farmacologia e cosmetologia, e para curso superior nas disciplinas de assistência farmacêutica, química farmacêutica, farmacologia, biologia, genética e fisiologia. Atualmente, atuando na assistência farmacêutica do SUS.
} 
resultados deste estudo possam ser fonte de conhecimento para muitos adolescentes e jovens que utilizam a CE, mudando a percepção sobre o seu uso e as consequências, principalmente levando a informação de que o mesmo ser deve ser utilizado quando não foi feito uso de anticoncepcionais ou preservativos durante o ato sexual, em casos de falha no método de rotina, uso de anticonceptivo inadequado e/ou ocorrências de violência sexual.

Palavras-chave: Pílula do dia Seguinte. Fármacos. Organismo da mulher. Anticonceptivo.

ABSTRACT: The emergency contraceptive (CE), popularly known as the "morning after pill" marketed in Brazil, is a method that differs from other contraceptives as it is used after unprotected sex, while the others prevent conception before and during sexual intercourse. This study aimed to provide the reader with a clear analysis of their use and effects, both desirable and undesirable, on the woman's body, due to the recurrent use of these contraceptives. Therefore, a theoretical review of the narrative type was carried out, based on researches and works published between the years 2011 to 202I. It is concluded with this study that there is a need for women to receive more information about indication, available methods, efficacy and fundamental safety measures in order to avoid an unwanted pregnancy and that the use of EC regularly can indeed cause health problems. It is expected that the results of this study can be a source of knowledge for many adolescents and young people who use EC, changing the perception of its use and consequences, mainly leading to the information that it should be used when it was not done. use of contraceptives or condoms during sexual intercourse, in cases of failure in the routine method, use of inadequate contraceptives and/or occurrences of sexual violence.

Keywords: Morning-after pill. Drugs. Woman's body. Contraceptive.

\section{INTRODUÇÃO}

A anticoncepção de emergência ou mais conhecido como contraceptivo de emergência (CE) é um método anticonceptivos feito a base de hormônios concentrados, que devem ser usados em um período curto de tempo após a relação sexual sem proteção, interrompendo a ovulação e migração dos espermatozoides. $O$ uso dos CE deve ser utilizado somente em ocorrências específicas (PAIVA; BRANDÃO, 2012; LIMA; SILVA; ADAMI, 2020). A indicação de seu uso é para quando não foi utilizado anticoncepcionais ou preservativo durante o ato sexual, em casos de falha no método em uso de rotina, uso de anticonceptivo inadequado e/ ou ocorrências de violência sexual (BRANDÃO et al., 2017). 
Conforme o Conselho Federal de Farmácia (20II), os métodos de contracepção de emergência não são considerados como abortivos, apesar de serem utilizados após a relação, antes da implantação do zigoto no útero, como uma medida ocasional e não como método contraceptivo de uso regular. Neste sentido, a falta de conhecimento sobre a farmacodinâmica do CE faz com que ocorram conclusões equivocadas sobre seus efeitos no organismo da mulher (OLSEN et al., 2018; PAIVA; COSTA, 2020).Os CE somente são efetivos quando ingeridos até 72 a 120 horas pós-coito.No entanto, estes terão maior efetividade quando administrados nas primeiras I2 horas, na opção de dose única (LACERDA, PORTELA; MARQUES, 2019).

Os fármacos de contracepção de emergência são popularmente conhecidos como "pílula do dia seguinte" (PDS) e possuem em sua composição hormônios muito similares com aqueles encontrados em outros contraceptivos de uso contínuo, porém com uma dosagem hormonal superior. Uma PDS é o mesmo que cerca da metade de uma cartela de anticoncepcional de uso regular (BRAMBILLA; RIECHEL; AMADEI, 2or6; MATSUOKA; GIOTTO, 2019). A ação do contraceptivo de emergência no organismo feminino prorroga, ou até mesmo dificulta, que os ovários liberem óvulos e, quando ocorre a liberação desses, não ocorre a fecundação, pois os óvulos alojam-se na parede do útero (LACERDA, PORTELA; MARQUES, 2019; MATSUOKA; GIOTTO, 2019).

Estima-se que no Brasil exista cerca de io milhões de mulheres que estejam expostas à gestação indesejada, em decorrência da falta de um método contraceptivo eficiente, uso inadequado dos anticoncepcionais ou até mesmo por falta de informação (PORTELA, 2015; VIEIRA; GERON, 2018). Vale ressaltar uma questão importante com relação a diferença socioeconômica, levando ao uso do fármaco de maneira irracional e inadequada, principalmente pelas adolescentes (MATSUOKA; GIOTTO, 2019).

Acredita-se que conhecer a farmacodinâmica dos CE mais utilizados, bem como falar sobre a relação da concentração e eficácia, além dos principais efeitos que podem causar, quando administrados incorretamente, poderá levar a população a uma melhor compreensão sobre esta opção de contracepção oferecida pelo mercado farmacêutico (COSTA et al., 2020). Nessa perspectiva, o objetivo desse estudo foi realizar uma revisão 
teórica do tipo narrativa que possa forneça a compreensão da farmacodinâmica dos CE, realizando uma análise sobre seu uso e efeitos desejáveis e indesejáveis no organismo da mulher, proveniente do uso recorrente desses contraceptivos.

\section{REVISÃO TEÓRICA}

\section{I Histórico, conceito e definição do $\mathrm{CE}$}

Os CE, também conhecidos como "pílula do dia seguinte" ou "pílula pós-coital", tratam-se de um procedimento contraceptivo que se distingue dos demais métodos anticoncepcionais, pois é usado após o ato sexual, enquanto que os demais previnem a não concepção antes e durante as relações sexuais (BRASIL, 20II; LEITÃ̃O, 20I6). Trata-se de um procedimento farmacológico que busca evitar a gravidez depois de relação sexual, sendo que esta pode ser sido sem proteção ou quando houve falha em algum dos demais métodos já usados (VIEIRA, GERON, 20I8; OLIVEIRA; OLIVEIRA, 2019).

Alguns estudos comprovam o uso indiscriminado pelas mulheres dos CE, mesmo que sua indicação seja somente parasituações adversas ou emergenciais, como já citadas anteriormente (BRAGA; SOUZA, 20I6; VIEIRA, GERON, 2018).

As pesquisas relacionadas aos CE foram iniciadas pelo médico canadense chamada Dr. Albert Yuzpe nos anos de 1960. O procedimento original tratava-se da combinação de estrogênio e progesterona, com o objetivo de evitar uma gravidez decorrente de abuso sexual (COSTA et al., 2020; LACERDA, PORTELA; MARQUES, 2019).

A partir do ano de 1970, o CE foi disponibilizado no mercado, primeiro na Hungria e em 1980 na China e Suécia (LEITÃO, 2016). Em 1996 foi regulamentado pelo Ministério de Saúde brasileiro e aprovado pela vigilância sanitária, sendo disponibilizado comercialmente mediante prescrição médica e incluído no mesmo ano nas normas técnicas de Planejamento Familiar (PF). No ano de 1998 passou a fazer parte das normas de violência sexual (BRASIL, 20II). No Brasil o CE era administrado através de uma combinação de pílulas anticoncepcionais orais comuns (dosagem combinada em maior quantidade) para que o resultado fosse atingido de maneira eficaz (BRAZ; FARIA; NABAS, 2014; BRANDÃO et al., 2017).

Comercialmente, a apresentação do comprimido de Levonorgestrel é o,75 mg. Dessa forma, para ministrar a contracepção de emergência é recomendado o uso de dois comprimidos de $0,75 \mathrm{mg}$ em uma única dose ou I comprimido a cada 12 horas (BRASIL, 20II; BRANDÃO et al., 2017; LACERDA, PORTELA; MARQUES, 2019).

O Levonorgestrel já é encontrado no comércio em um único comprimido de 1,5 mg, nesse caso usuária fará uso dose única (LEITÃO, 20ı6; BRAGA; SOUZA, 2016). Para que ocorra uma maior eficácia, recomenda-se que a administração do comprimido ocorra o mais cedo possível, após uma relação sexual desprotegida (PAIVA; BRANDÃO, 20I2; BRAGA; SOUZA, 2016).

O serviço público na busca de ampliar a oferta dos CE permitiu a prescrição por enfermeiros, porém somente em situações especiais, através da Lei n.ำ 7.498/86, artigo II, 
item II, $\S 3^{\circ}$ “os profissionais de enfermagem podem receitar medicamentos anteriormente definidos em programas de saúde pública". No entanto, é essencial que esse profissional tenha sido capacitado tecnicamente de acordo com o protocolo institucional. Além do mais, a prescrição deve ter o acompanhamento de um médico (BRASIL, 2oII). Inicialmente o método foi ofertado no regime Yuzpe, ou seja, pela combinação de pílulas anticoncepcionais orais comuns, à base de etinilestradiol e levonorgestrel(BRANDÃO et al., 2017).

O Ministério da Saúde, em 2003, passou a disponibilizar os CE de forma mais ampla e os serviços de saúde passaram a garantir às mulheres o método por intermédio de indicações estabelecidas, ou seja, situações em que não foi usado método anticoncepcional tradicional, em provável falha de método anticoncepcional regular e casos de estupro (SOUSA; CIPRIANO, 2019; BRANDÃO et al., 2017).

A partir de então, as pílulas foram tomadas de forma combinada para atingir tal objetivo, porém provocavam diversos efeitos adversos, como náusea, vômito e dor de cabeça. Anos depois, em 1990, foi criado um medicamento composto por um único hormônio, a progesterona, que diminuiu os efeitos adversos e aumentaa efetividade (BRANDÃO et al., 2017; RORIZ; RIBEIRO, 2016, PAIVA; COSTA, 2020).

Em 2012, foi divulgado um Protocolo para Utilização do Levonorgestrel na Anticoncepção Hormonal de Emergência, abrindo um leque de possibilidades de distribuição do medicamento na atenção básica de saúde, por meio da prescrição do médico ou do enfermeiro, nas unidades básicas de saúde (UBS), em unidades de pronto-atendimento e centros de saúde e unidades da Estratégia de Saúde da Família (ESF) (BRASIL, 2012; BRANDÃO et al., 2017).

Atualmente, conforme o Ministério da Saúde, existem duas maneiras aceitas e recomendadas dos CE, que é o Yuzpe e o Levonorgestrel (LNG). Sendo que a primeira consiste na administração de pílulas anticoncepcionais combinadas, compostas de estrogênio e progestogênio sintéticos, e a segundatrata-se de uma composição exclusiva de progestogênio isolado, em uma dose total de I,5 mg (LEITÃO, 2016).

\subsection{Métodos disponíveis no mercado e sua eficácia}

Os métodos de CE disponíveis atualmente no mercado são o LNG I,5 mg, que se trata de um progestágeno, sendo indicado seu uso até 72 horas após a relação sexual desprotegida. O outro método é Yuzpe, uma combinação de progestágeno e estrogênio em altas dosagens ( $500 \mu \mathrm{g}$ de LNG + 10o $\mu \mathrm{g}$ de Etinilestradiol, em duas tomadas com intervalo de $12 \mathrm{~h}$ ), já mencionado anteriormente. Devido ao fato que os efeitos colaterais serem maiores, as pílulas são utilizadas somente na falta do Levonorgestrel no mercado ou unidades de saúde (BRASIL, 20Ir; OLIVEIRA; BURCI, 2019).

Outro método disponível é o dispositivo intrauterino (DIU) de cobre, inserido em até 5 dias após relação desprotegida ou ovulação. Já o método com a utilização do Acetato de Ulipristal $30 \mathrm{mg}$, um modulador do receptor de estrogênio, indicado até $120 \mathrm{~h}$ após relação sexual desprotegida. Por último, o Mifepristone, que é um modulador do receptor 
de progesterona que tem sido estudado atualmente, mas está disponível apenas na China e no Vietnã (CANOVA; CARUSO; POLI, 2012).

Vale ressaltar que esses CE devem ser empregados de modo ocasional e nas situações específicas acima citadas, pois além de não fornecer proteção contra Infecções Sexualmente Transmissíveis (IST), existem outros métodos contraceptivos mais eficazes para uso contínuo (LEITÃO, 2016; COSTA et al., 2020).

Em relação à eficácia, como dos demais contraceptivos, pouco se encontra na literatura estudos sobre a eficácia do uso dos CE e dos estudos existentes, a maioria apresenta resultados conflitantes.

\subsection{Mecanismos de ação e a relação da concentração do CE}

Os CE são o único método que pode ser utilizado na prevenção da gravidez após a relação sexual,atuando na interrupção do ciclo reprodutivo da mulher, alterando dessa forma, os fenômenos biológicos que resultariam na gestação. Eles retardam a ovulação e prejudicam a locomoção do espermatozoide no útero, assim, sua ação e reação são anteriores a fecundação (BRASIL, 20II; ALANO et al., 2012).

Um dos métodos de CE mais utilizados é o Levonorgestrel que é metabolizado e absorvido no intestino e quando cai na corrente sanguínea, age na inibição ou retardo da ovulação devido à modificação da mutabilidade tubária, a inversão das contrações e do batimento das fímbrias e na alteração da movimentação dos espermatozoides, pelo aumento do $\mathrm{pH}$ do fluido uterino e da viscosidade do muco cervical (CAVALCANTE et al., 2016; BRAZ; FARIA; NABAS, 2014).

Esse fármaco age antes da fecundação do espermatozoide no óvulo, sendo que sua ação não interrompe uma gravidez, pois sua ingestão deve ocorrer antes das 72 horas após o ato sexual, onde sua principal função é evitar uma gravidez indesejada (HEVIA, 2012, LEITÃO, 2016). Esse intervalo sugerido para administração se dá pelo fato de a geração de um embrião só ocorrer aproximadamente 120 horas depois da relação sexual (BRANDÃO et al., 2017; PORTELA, 2015). 
Entre as reações adversas no organismo feminino, os mais observados são vômitos, sangramento uterino irregular, náuseas, antecipação ou atraso da menstruação, aumento da sensibilidade mamária, retenção hídrica e cefaleia (SOUSA; CIPRIANO, 2019).

Uma das questões que preocupava o Ministério da Saúde e alguns pesquisadores era sobre a existência do efeito abortivo pelo uso dos CE, (BATAGLIÃO; MAMEDE, 2oIr; OLIVEIRA; BURCI, 2019), mas não existem conclusões concretas que esses fármacos possuem esse efeito. No entanto, quando esses fármacos são usados em altas doses, apresentam potencial risco de formação de coágulos sanguíneos e de ocorrer falha na medicação, além de causar náuseas e vômitos muito fortes a ponto de serem ineficazes. Também é recomendado que antes da administração da "pílula do dia seguinte" seja descartada a gravidez por meio de testes (PORTELA, 2015).

\subsection{Contraceptivos de emergência e os riscos à saúde da mulher}

Estudos apontam que dos métodos mais vendidos no mercado, o Yuzpe é que vem apresentando uma incidência maior de vômitos e náuseas entre as pacientes, comparado ao Levonorgestrel. As pacientes que fizeram uso do acetato de Ulipristal parecem que eram mais propensas ao retorno menstrual antes do período previsto, comparado as que utilizaram de Levonorgestrel. As pacientes que fizeram uso de Levonorgestrel são bem mais propensas a antecipação da menstruação do que as do Yuzpe. De todos os CE, em relação a dores abdominais há uma maior incidência entre as usuárias do DIU (VALVERDE; OLIVEIRA, 20I4; CANOVA; CARUSO; POLI, 2020).

O Levonorgestrel, entre os CE, é considerado como um procedimento de grande eficácia, porém ressalta-se que quando este é utilizado por períodos longos, dosagens altas ou de forma impensada pode provocar sérios problemas à saúde da mulher. Entre os problemas, relata-se a possibilidade de desenvolver câncer de colo uterino e de mama, além da não eficácia terapêutica do fármaco, promovendo uma gravidez indesejada e levando a infertilidade. Além disso, não oferece proteção para Infecções Sexualmente Transmissíveis (IST) e Vírus da Imunodeficiência Humana (HIV), a Síndrome da Imunodeficiência Adquirida (AIDS). Assim, é indicado que a utilização dos CE seja feita 
com cautela e de preferência, por meio de prescrição médica (LACERDA; PORTELA, 2019; COSTA et al.; 2020).

Adicionalmente, a literatura relata que o uso de Levonorgestrel pode acarretar outros efeitos à saúde das pacientes, entre os mais corriqueiros pode-se citar cefaleias, aumento da pressão arterial, inchaço, sensação de falta de ar e alteração no ciclo menstrual (MATSUOKA; GIOTTO, 2019; SOUSA; CIPRIANO, 2019). Não existem estudos que comprovem caso de morte ou complicações ligadas aos CE (BRANDÃO et al., 2017; COSTA et al.; 2020).

\section{CONCLUSÕES}

A partir da leitura dos trabalhos selecionados, acredita-se que há necessidade de que as mulheres recebam maiores informações sobre indicação, métodos disponíveis, eficácia e segurança fundamentais a fim de evitar uma gravidez indesejada e que o uso de CE regularmente pode sim causar problemas de saúde.

Mesmo que não tenha sido o foco desse estudo, cabe aqui elencar ainda sobre a importância dos profissionais de saúde em implantar junto as unidades básicas de saúde, ações de educação em saúde sexual sobre a temática, direcionada preferencialmente a população feminina, adolescentes e jovens. Buscando promover a conscientização das mesmas à utilização dos métodos de CE de maneira correta, evitando assim possíveis consequências a saúde da mulher.

Espera-se que os resultados deste estudo possam ser fonte de conhecimento há muitas adolescentes e jovens que utilizam a CE, mudando sua percepção sobre o uso e as consequências e que o mesmo, só pode ser utilizado nos casos específicos mencionados nos inúmeros estudos apresentados no decorrer dessa pesquisa.

Ainda, acredita-se que mesmo frente suas limitações, essa pesquisa possam abrir novos caminhos para futuros estudos na área da saúde sobre os métodos contraceptivos, além de ser suporte científico para novos trabalhos acadêmicos sobre o tema. 


\section{REFERÊNCIAS BIBLIOGRÁFICAS}

AlANO, G. M; COSTA, L. N; MIRANDA L. R; GALATO, D. Conhecimento, consumo e acesso à contracepção de emergência entre mulheres universitárias no sul do estado de Santa Catarina. Ciênc. Saúde Coletiva. 2012; 17(9), p. 2397-2404.

BRAMBILlA, A., RIECHEL, T., AMADEI, J. L. Contracepção de emergência e universitárias da área da saúde. Revista de Saúde e Educação - SUSTINERE, v. 4, n. 2, p. 253-264, 2016.

BATAGLIÃO,E.M.L.;MAMEDE,E.F.C.ConhecimentoeutilizaçãodaContracepçãode emergência por acadêmicos de enfermagem. Esc Anna Nery (impr.) abr-jun;15(2), p.284290,2011.

BRAGA, A.P. C., SOUZA, A.C. Efeitos do uso da Contracepção de Emergência: Revisão de Literatura, Monografia (graduação em Biomedicina), Faculdade de Ciências da Educação e Saúde - FACES, Brasília- DF, p. 22. 2016.

BRANDÃO, E. R., CABRAL, C. S; VENTURA, M., PAIVA, S. P; BASTOS, L. L., OLIVEIRA, N. V. B. V; SZABO, I. "Bomba hormonal": os riscos da contracepção de emergência na perspectiva dos balconistas de farmácias no Rio de Janeiro, Brasil.Cad Saúde Pública, p. II. 2017.

BRANDÃO, E. R.; CABRAL, C. DA S.; VENTURA, M.; PAIVA, S. P.; BASTOS, L. L.; DE OLIVEIRA, N. V. B. V. \& SZABO, I. Os perigos subsumidos na contracepção de emergência: moralidades e saberes em jogo. Horizontes Antropológicos, v. 23, n. 47, p. 131- I6I. 2017.

BRANDÃO, E. R; CABRAL, C. S. Contracepção de emergência no Brasil: desafios para a assistência farmacêutica. Saúde e Sociedade, São Paulo, v. 26, n. 4, p. I087-1092, 2017. Disponível em: http://www.scielo.br/pdf/sausoc/v26n4/1984-0470-sausoc-26-o4-ı87.pdf. DOI: 10.1590/so104-I290201700000o.

BRAZ, A. G.; FARIA J. G.; NABAS, J. M. A. B. B. Levonorgestrel: considerações sobre o uso da contracepção de emergência - orientações básicas, 2014. 
BRASIL. Ministério da Saúde. Protocolo para utilização do levonorgestrel na anticoncepção hormonal de emergência. Brasília, 2012.

BRASIL. Ministério da saúde. Anticoncepção de Emergência: perguntas e respostas para profissionais de saúde. Brasília-DF, 2011.

CANOVA, R. S; CARUSO, F. B; POLI, M. E. H. Contracepção de Emergência: $\begin{array}{llllll}\text { Indicações } & e & \text { Métodos, } & \text { 202I, } & \text { p. }\end{array}$ https://docs.bvsalud.org/biblioref/2018/o4/882343/contracepcao-de-emergenciaindicacoes-e-metodos.pdf acesso em: maio de 2021.

CARVALHO, A. L. M. N; SILVEIRA, N. J. Automedicação Em Itumbiara-Go - Análise Química E Qualitativa Dos Riscos E Benefícios Monografia (Licenciatura em Química), Instituto Federal de Educação, Ciência e Tecnologia de Goiás. p. 51. 2017. https://repositorio.ifg.edu.br/handle/prefix/269 acesso em: abril de 2021.

CAVAlCANTE, M. De S.; SOARES, M. A.; FEIJÓ, C. M.; FONTEleS, M. M. de F. Perfil de utilização de anticoncepcional de emergência em serviços de atendimento farmacêutico de uma rede de farmácias comunitárias. Revista Eletrônica de Farmácia, v. I3, n. 3, p. 131-139, 2016.

COSTA, R. J. F, et al., O uso de contraceptivos de emergência por estudantes de uma instituição de ensino superior de Belém, Pará, Rev. Bra. Edu. Saúde, REBES, v. ı, n.4, p. I24-130, out-dez, 2020 ISSN 2358-2391 Pombal, PB, 2020.

DE’ luCiA R., Planeta C. S., GAllaCCi, M., AVEllaR M. C. W., Filho, R. M. O. FARMACOLOGIA INTEGRADA Uso Racional de Medicamentos, Ricardo De Lúcia (org), 5ª , vol. I,São Paulo: Clube de Autores, p. 44I, 2014.

HEVIA, M. The legal status of emergency contraception in Latin America.International Journal of Gynecology and Obstretrics. London, II6 (I), p. 87-90, 2012.

LEITÃ O, K, R. S. Conhecimento e utilização da anticoncepção de emergência entre os acadêmicos da Universidade Federaldo Maranhão, Monografia (Graduação de Enfermagem), Universidade Federal do Maranhão, UFMA, p.63, 2016. 
LACERDA, J. O. S.; PORTELA, F. S.; MARQUES, M. S. O Uso Indiscriminado da Anticoncepção de Emergência: Uma Revisão Sistemática da Literatura. ID online Revista de Psicologia, v. 13, n. 43, p. 379-386, 2019.

LIMA, F.C.F; SILVA, L.C.M; ADAMI, E.R. Uso de contraceptivos de emergência universitárias da área da saúde do curso de farmácia. Revista UNIANDRADE, v.21, n.2, p.82-88, 2018.

MATSUOKA, J. S..; GIOTTO, A. C. Contraceptivo de emergência, sua funcionalidade e a atenção farmacêutica na garantia de sua eficácia. Revista de Iniciação Científica e Extensão, v. 2, n. 3, p. 154-162, I6 ago. 2019.

NUNES, I. K. C. A Importância do Estudo do Metabolismo nos Estágios Iniciais de Desenvolvimento de Fármacos Rev. Virtual Quim. 7 (2), 649-662, 2015. http://rvqsub.sbq.org.br/index.php/rvq/article/view/ro83. acesso em: abril de 2021.

OLIVEIRA, M.I.C.; OLIVEIRA, V. B. Avaliação quantitativa da dispensação de contraceptivos de emergência na região de Curitiba, PR, Brasil, entre 2012 e 2014. InfarmaCiênciasBrazilianJournalofForensicSciences, Medical Law and Bioethics 8(3):165177, 2019.

OLIVEIRA, A. P. R.; BURCI, L. M. Percepção Bioética dos Enfermeiros na Administração e/ou Orientação do Uso do Contraceptivo de Emergência, BrazilianJournalofForensicSciences, Medical Law andBioethics, v. 8, n. 3, p. 165-177, 2019. OLSEN, J.M.; LAGO, T.D.G;.KALCKMANN, S.; ALVES, M.C.G.P.; ESCUDER, M.M.L. Práticas contraceptivas de mulheres jovens: inquérito domiciliar no Município de São Paulo, Brasil. Cad Saúde Pública 2018; 34:e ooorg617.

PAIVA, S; BRANDÃO, E.R, Contracepção de emergência no contexto das farmácias: revisão crítica de literatura. Physis: Revista de Saúde Coletiva. vol. 22 no.I Rio de Janeiro 2012, p. 17-34. https://doi.org/10.1590/So103-73312012000100002 acesso em: abril de 2021.

PORTElA, C. G. Uso Discriminado Da Pílula Do Dia Seguinte. Monografia (Graduação em Farmácia), FAEMA - Faculdade de Educação e Meio Ambiente, Ariquemes - RO, 2015, p. 29. http://repositorio.faema.edu.br/ acesso em: maio de 2021. 
PRABAKAR, I, WEBB, A. Contracepção de emergência. BrMed J 2012;344:6I64 .doi: https://doi.org/Io.I136/bmj.er492. acesso em: abril de 2021.

RORIZ, L.; RIBEIRO, M. L. P. da C. A pílula do dia seguinte: um estudo sobre a percepção ético-moral de acadêmicas de enfermagem da FACESA. Revista de Divulgação Científica Sena Aires. 2016; 5(I), p. 63-9.

SOUSA, L. G., CIPRIANO, V. T. F. Contraceptivo oral de emergência: indicações, uso e reações adversas. Revista Eletrônica Acervo Saúde, n. 22, p. 665- 665, 2019.

VALVERDE K. C. L, OLIVEIRA, A. V. Adolescentes e jovens e a contracepção de emergência: Revisão integrativa da literatura. Rev. de Divulgação Científica Sena Aires. 2015; 3(2): 185-94.

VIEIRA, G, V., GERON, V. L. M. Fatores associados ao uso abusivo do contraceptivo de emergência e seus efeitos indesejados em acadêmicas da área da saúde de uma faculdade de Ariquemes Rondônia. Monografia (Graduação em Farmácia), Faculdade de Educação e Meio Ambiente, Ariquemes Rondônia, p. 34, 2018. 